\title{
Higher-Order Circularity based I/Q Imbalance Compensation in Direct-Conversion Receivers
}

\author{
Fanglin Gu, Shan Wang, and Jibo Wei \\ College of Electronic Science and Engineering \\ National University of Defense Technology \\ Changsha, China 410073 \\ Email: gu.fanglin, chinafir, wjbhw@ nudt.edu.cn
}

\author{
Wenwu Wang \\ Department of Electronic Engineering \\ University of Surrey \\ Guildford GU2 7XK, U.K. \\ Email: w.wang@surrey.ac.uk
}

\begin{abstract}
In-phase and quadrature-phase (I/Q) imbalance is a critical issue limit the achievable operating signal-to-noise ratio (SNR) at the receiver in direct conversion architecture. In recent literatures, the second- and fourth-order circularity property of communication signals have been used for designing compensator to eliminate the $I / Q$ imbalance. In this paper, we investigate whether moment circularity of an order higher than four can be used in receiver $I / Q$ imbalance compensation. It is shown that the sixth-order moment $\mathrm{E}\left[\mathrm{z}^{4} \mathrm{z}^{* 2}\right]$ is a suitable statistic for measuring the sixth-order circularity of representative communication signals such as $M$-QAM and $M$-PSK with $M>2$. Two blind algorithms are then proposed to update the coefficients of $I / Q$ imbalance compensator by restoring the sixth-order circularity of the compensator output signal. Simulation results show that the new proposed methods based on sixth-order statistic converges faster or gives lower steady-state variance than the reference methods that are based on second- and fourth-order statistics.
\end{abstract}

\section{INTRODUCTION}

In recent years, direct conversion receiver has attracted many research attention due to its low power consumption and low implementation cost. However, it suffers from serious in-phase and quadrature-phase (I/Q) imbalance, which arises from amplitude and phase mismatches of the two physical analog signal paths of the I/Q receiver. The I/Q imbalance can severely limit the achievable operating signal-to-noise ratio (SNR) at the receiver, and consequently, the supported constellation sizes and data rates. Hence, in order to produce the best possible advantages of direct conversion receiver, it is necessary to compensate the I/Q imbalance. There have been many reports in the literature on the compensation of the $\mathrm{I} / \mathrm{Q}$ imbalance $[1,2,3,6,7,8]$. The most intuitive approach for an estimation of the I/Q imbalance parameters is to feed the I/Q mixer with delicate calibration or training signals [1-3]. However, such techniques are limited to a certain class of communication standards with presumed pilots.

In order to cope with receiver I/Q imbalance in a wide range of present and future communication standards, hence, we mainly focus on blind compensation of I/Q imbalances in the general wideband receiver context. The leading principle for blindly designing I/Q imbalance compensator is to utilize the so-called circularity or properness feature [4] of complex communication waveforms. Preliminary results with frequencyindependent I/Q imbalances have been presented by Valkama et al. [5] in a basic single-channel receiver context and in a general I/Q receiver context, which form the starting point of this paper. These results were extended to more general cases in [6]. It has been shown that, the circularity-based algorithms can be performed blindly and directly on the downconverted complex signal, regardless the linear distortion and frequency offset of the channel (as long as the circularity assumption still holds). This is advantageous from the practical system implementation point of view. In these literatures, however, the mirror-frequency interference resulting from the I/Q imbalance is mitigated by resorting to the second-order circularity property of communication signals.

Recently, it has been shown in [7] that the fourth-order circularity can also been used for I/Q imbalance compensation, and in comparison with the method based on second-order circularity, the method based on fourth-order circularity can achieve higher image rejection value and faster convergence. It is natural to ask whether moment circularity of an order higher than four can be exploited for designing receiver I/Q imbalance compensator. If so, are there any general conclusions for the choice of higher order circularity? In this paper, we investigate whether the moment circularity of an order higher than four can be applied in receiver I/Q imbalance compensation. To this end, the main contributions of this paper are as follows.

- We investigate the feasibility of using the higher order circularity for designing I/Q imbalance compensator. It is verified that the moment $\mathrm{E}\left[\mathrm{z}^{4} \mathrm{z}^{* 2}\right]$ is a suitable measure of circularity for a wide range of variables and signals encountered in communications.

- Based on the concept of sixth-order circularity, a Newton's algorithm and its stochastic form are proposed to obtain the desired filter coefficient in I/Q imbalance compensator.

\section{PROBlem Formulation}

Considering the mixer I/Q imbalance caused by an I/Q local oscillator, the received baseband signal is generally expressed in a widely-linear form as [1]

$$
x(n)=K_{1} z(n)+K_{2} z^{*}(n)
$$

with $K_{1}=(1 / 2)(1+g \exp (-j \phi))$ and $K_{2}=(1 / 2)(1-$ $g \exp (j \phi))$ and $g$ and $\phi$ representing the amplitude and phase mismatch coefficients, respectively, where $j$ denotes the 
imaginary unit and $\exp ($.$) denotes exponential operation. The$ ideal complex envelope $z(n)$ is the received signal without I/Q imbalance, consisting of the channel-distorted received signal and additive noise, and $n$ is discrete time index. The image rejection ratio (IRR) which reflects image rejection in $\mathrm{RF}$ transmitters and receivers, is defined as [6]

$$
\operatorname{IRR}=\frac{\mathrm{E}\left[\left|K_{1} z(n)\right|^{2}\right]}{\mathrm{E}\left[\left|K_{2} z^{*}(n)\right|^{2}\right]}=\frac{\left|K_{1}\right|^{2}}{\left|K_{2}\right|^{2}}
$$

where $\mathrm{E}[$.$] is ensemble mean and |$.$| is the Frobenius norm.$ The I/Q imbalance values typically range between $1-10 \%$ for the gain imbalance, and 1-10 degrees for the phase imbalance, given IRRs in the range $20-40 \mathrm{~dB}$ [5].

The aim of I/Q imbalance compensation is to mitigate the interference caused by its conjugate counterpart. Obvious, the IRR value of the post-compensation is higher, the performance of I/Q imbalance compensator is better. Ideally, the IRR value would approach infinite with perfect I/Q imbalance compensation.

\section{HigheR-ORDER CiRCUlARITY-BASED I/Q COMPENSATOR}

For the current problem, we assume that the received signal without I/Q imbalance is circular or at least circular at some order moments, and is then made noncircular by the I/Q imbalance. In the $\mathrm{I} / \mathrm{Q}$ imbalance compensation stage, the signal is then forced circular again through adaptive filtering techniques to cancel the effects of I/Q imbalance. Thus, for a statistic to be a candidate for the adaptive learning rule, it should vanish with typical communications signals, additive noise, and sums of them, but become non-zero if the received signals corrupted by I/Q imbalance.

To that end, we firstly analyze the circularity of the Random Variable (RV) $z$ with some common probability distributions encountered in communications. In particular, we investigate the cases when $z$ takes its values from the common complexvalued alphabets $M$-QAM and $M$-PSK, and when $z$ is complex-circular Gaussian. Then, we design an I/Q imbalance compensator by resorting to the sixth-order circularity property of the communication signals.

\section{A. Nth-Order Circularity Moments of Some Communication Signals and Gaussian Noise}

For a complex-valued $\mathrm{RV} z$, strict circularity means that $z$ and $e^{j \phi} z$, for any $\phi \in[0,2 \pi)$, have the same probability distribution. Intuitively, a $\mathrm{RV} z$ is circular if its statistical properties are "invariant under a rotation". However, strict circularity is difficult to measure. Hence, in practice, a weaker form of circularity called moment circularity or $N$ th-order circularity is usually employed. $N$ th-order circularity is defined as $z$ and $e^{j \phi} z$, for any $\phi \in[0,2 \pi)$, having equal moments up to order $N$. This implies, that for an $N$ th order circular random variable, its only non-zero moments up to order $N$ are the ones with an equal number of non-conjugated and conjugated terms, since only in these cases the phase terms cancel out.
Nevertheless, even if for the $N$ th-order circularity, it is still difficult to measure and use. The main reason is that there are $(n+1)$ different moments $\mathrm{E}\left[z^{* n-p} z^{p}\right]$ for complex RVs for a given order $n$. Hence, in practice, a representative statistic is usually chosen from the $(n+1)$ different moments to measure the circularity according to the distribution of signals. Until now, the circularity moments of an order higher than two are rarely discussed in the literature. To the best of our knowledge, only the fourth-order circularity moment $\mathrm{E}\left[z^{3} z^{*}\right]$ is used to measure the degree of circularity for a wide range of communication signals [7]. It would be interesting to see how the circularity moment of an order higher than four performs. To this end, we derive a rule to choose the representative statistic for measuring the sixth-order circularity. A similar rule can be easily derived for measuring the general higherorders.

According to the definition of circularity in terms of moments, the conditions of circularity for sixth-order circularity are

$$
\begin{gathered}
m_{z}(6,0)=\mathrm{E}\left[z^{6}\right]=0 \\
m_{z}(5,1)=\mathrm{E}\left[z^{5} z^{*}\right]=0 \\
m_{z}(4,2)=\mathrm{E}\left[z^{4} z^{* 2}\right]=0
\end{gathered}
$$

The statistics in (3a), (3b) and (3c) will herein be called the sixth-order circularity moments. In general, the conditions for circularity can be similarly extended to higher-order moments.

1) M-QAM and M-PSK Symbol Sequences: It has been shown that the statistic $\mathrm{E}\left[\mathrm{z}^{4}\right]$ will not vanish for $M$-QAM symbol sequences with equally probable symbols. For example, for 4-QAM and 16-QAM alphabets and equally probable symbols, the normalized statistic $\mathrm{E}\left[\mathrm{z}^{4}\right] / \mathrm{E}\left[|\mathrm{z}|^{2}\right]^{2}$ equals -1 and -0.68 , respectively. Owing to $E\left[z^{5} z^{*}\right]=E\left[|z|^{2} z^{4}\right]$ and $|z|^{2}>0$, it is straightforward to derive that $\mathrm{E}\left[\mathrm{z}^{5} \mathrm{z}^{*}\right]$ will not vanish for $M$-QAM symbol sequences with equally probable symbols. For example, for 4-QAM and 16-QAM alphabets and equally probable symbols, the normalized statistic $\mathrm{E}\left[\mathrm{z}^{5} \mathrm{z}^{*}\right]$ equals -8 and -1320 , respectively. On the one hand, note that $\mathrm{E}\left[\mathrm{z}^{4} \mathrm{z}^{* 2}\right]=\mathrm{E}\left[|\mathrm{z}|^{4} \mathrm{z}^{2}\right]$ and $\mathrm{E}\left[\mathrm{z}^{2}\right]=0$, the conditions in (3a) and (3c) will indeed be satisfied by all the $M$-QAM symbol sequences containing equally probable symbols. These can also be shown by direct substitution. On the other hand, $M$ PSK alphabets with $M>2$ satisfy not only (3a) and (3c), but also (3b).

From the above analysis, we can see that both the statistics $\mathrm{E}\left[\mathrm{z}^{4} \mathrm{z}^{* 2}\right]$ and $\mathrm{E}\left[\mathrm{z}^{6}\right]$ can be used to measure the sixth-order circularity. However, for our problem, the rule to choose the statistic is determined by the performance of the adaptive learning rule for I/Q compensation, of which the convergence rate and post-compensation IRR are critical. The convergence rate of the adaptive learning rule is related to the gradient of the statistics with respect to the gain and phase imbalance factor. Generally, the adaptive learning rule based on the statistic which possesses steeper gradient with respect to the gain and phase imbalance factor, will have faster convergence rate. Fig. 1 (left) shows the surfaces of the statistics $E\left[z^{4} z^{* 2}\right]$ 

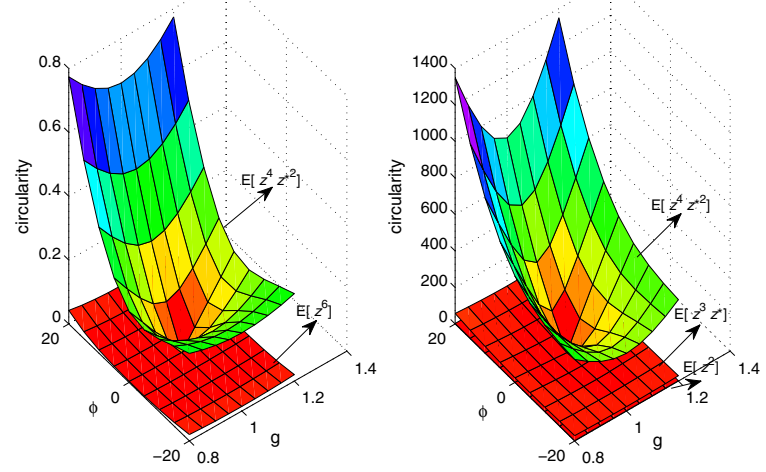

Fig. 1. The surface of statistics with respect to the gain imbalance factor $g$ and phase imbalance factor $\phi$.

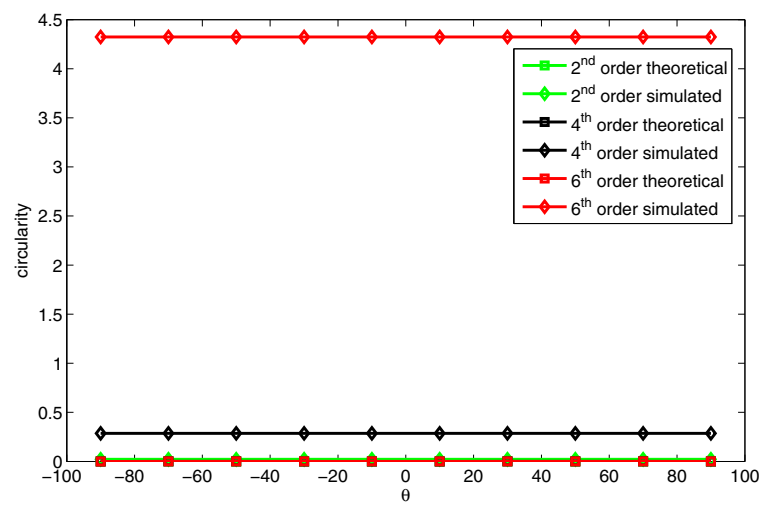

Fig. 2. The influence of phase shift on the circularity of a 16-QAM signal.

and $\mathrm{E}\left[\mathrm{z}^{6}\right]$ with respect to gain imbalance factor $g$ and phase imbalance factor $\phi$ for 16-PSK. In comparison with the statistic $E\left[z^{6}\right]$, the statistic $E\left[z^{4} z^{* 2}\right]$ has a steeper gradient with respect to the gain and phase imbalance factor. Hence, $\mathrm{E}\left[\mathrm{z}^{4} \mathrm{z}^{* 2}\right]$ is more preferable than $\mathrm{E}\left[\mathrm{z}^{6}\right]$ as the sixth-order circularity to be adopted in our algorithm. Fig. 1 (right) shows the surfaces of the statistics $E\left[z^{2}\right], E\left[z^{3} z^{*}\right]$ and $E\left[z^{4} z^{* 2}\right]$ with respect to gain imbalance factor $g$ and phase imbalance factor $\phi$ for 16-QAM. Among the referred three statistics, the statistic $\mathrm{E}\left[\mathrm{z}^{4} \mathrm{z}^{* 2}\right]$ also has the steepest gradient with respect to the gain and phase imbalance factor. It can be inferred that the adaptive learning rule based on the sixth-order moment $\mathrm{E}\left[\mathrm{z}^{4} \mathrm{z}^{* 2}\right]$ has the fastest convergence rate.

Another issue needs to be pointed out is that any phase shift $\theta \in[-\pi / 2, \pi / 2)$ has no influence on the statistics $\mathrm{E}\left[\mathrm{z}^{2}\right]$, $E\left[z^{3} z^{*}\right]$ and $E\left[z^{4} z^{* 2}\right]$ as shown in Fig. 2. This implies that the output of the circularity-based I/Q compensator has phase ambiguity. Therefore, techniques such as the MultiModulus Algorithm (MMA) [11] are needed to eliminate the phase ambiguity.

2) Gaussian Random Variables: For Gaussian RVs, secondorder circularity implies strict circularity and hence moment circularity of all orders [4]. Therefore, second-order circular Gaussian RVs, such as the complex envelope of the additive noise in RF front-ends, will satisfy conditions (3a-3c).

3) Sum of Two Nth-Order Circular RVs: For the sum of two mutually independent, zero-mean, sixth-order circular random variables $z_{1}$ and $z_{2}$, we get

$$
\begin{aligned}
& m_{z_{1}+z_{2}}(6,0)=m_{z_{1}}(6,0)+m_{z_{2}}(6,0) \\
& m_{z_{1}+z_{2}}(5,1)=m_{z_{1}}(5,1)+m_{z_{2}}(5,1) \\
& m_{z_{1}+z_{2}}(4,2)=m_{z_{1}}(4,2)+m_{z_{2}}(4,2)
\end{aligned}
$$

meaning that the sum of two sixth-order circular (noncircular) random variables remains circular (noncircular), except in the trivial cases of $m_{z_{1}}(6,0)=-m_{z_{2}}(6,0), m_{z_{1}}(5,1)=$ $-m_{z_{2}}(5,1)$ and $m_{z_{1}}(4,2)=-m_{z_{2}}(4,2)$.

In conclusion, the statistic $\mathrm{E}\left[z^{4} z^{* 2}\right]$ appears to be a plausible measure of circularity for a wide range of practical communications signals. For the rest of the paper, our focus will be on this statistic, and we will call the random variables which satisfy (3c) as sixth-order circular RVs.

\section{B. Sixth-Order Circularity-based I/Q Compensator}

The I/Q imbalance compensator is given as [6]

$$
y(n)=x(n)+w x^{*}(n)
$$

where $w$ denotes the compensator coefficient to be estimated. For simplicity, the compensation filter $w$ is assumed singletap. Nevertheless, the compensation filter can also be multitap filter, but it is beyond scope of this paper. In this case, the desired solution to $w$ can be easily obtained as

$$
w_{d}=-\frac{K_{2}}{K_{1}^{*}}
$$

if the imbalance coefficients $K_{1}$ and $K_{2}$ are known. However, there is little knowledge about these imbalance coefficients in practice.

Now, under the assumption that $z(n)$ is sixth-order circular and $K_{1}, K_{2} \neq 0$, by inserting (1) into (3c), it is straightforward to obtain the moment $m_{x}(4,2)$ of the received signal interfered by $\mathrm{I} / \mathrm{Q}$ imbalance as

$$
\begin{aligned}
& m_{x}(4,2)=4 K_{1} K_{2}\left(\left|K_{1}\right|^{4}+3\left|K_{1}\right|^{2}\left|K_{1}\right|^{2}+\left|K_{2}\right|^{4}\right) m_{z}(3,3) \\
& \quad+2\left(K_{1}^{3} K_{2}^{*}\left|K_{1}\right|^{2}+2 K_{1}^{3} K_{2}^{*}\left|K_{2}\right|^{2}\right) m_{z}(5,1) \\
& \quad+2\left(K_{1}^{*} K_{2}^{3}\left|K_{1}\right|^{2}+2 K_{1}^{*} K_{2}^{3}\left|K_{2}\right|^{2}\right) m_{z}^{*}(5,1) \\
& \quad+K_{1}^{4} K_{2}^{* 2} m_{z}(6,0)+K_{1}^{*} K_{2}^{3} m_{z}^{*}(6,0) \neq 0
\end{aligned}
$$

The objective in the forthcoming compensator developments is to restore the sixth-order circularity of the compensator output signal (5), i.e., to make

$$
m_{y}(4,2)=0
$$


Inserting (1) into (5) and evaluating the statistic $m_{y}(4,2)$, we get

$$
\begin{aligned}
& m_{y}(4,2)=4 K_{1}^{\prime} K_{2}^{\prime}\left(\left|K_{1}^{\prime}\right|^{4}+3\left|K_{1}^{\prime}\right|^{2}\left|K_{2}^{\prime}\right|^{2}+\left|K_{2}^{\prime}\right|^{4}\right) m_{z}(3,3) \\
& \quad+2\left(K_{1}^{\prime 3} K_{2}^{\prime *}\left|K_{1}^{\prime}\right|^{2}+2 K_{1}^{\prime 3} K_{2}^{\prime *}\left|K_{2}^{\prime}\right|^{2}\right) m_{z}(5,1) \\
& \quad+2\left(K_{1}^{\prime *} K_{2}^{\prime 3}\left|K_{1}^{\prime}\right|^{2}+2 K_{1}^{\prime *} K_{2}^{\prime 3}\left|K_{2}^{\prime}\right|^{2}\right) m_{z}^{*}(5,1) \\
& \quad+K_{1}^{\prime 4} K_{2}^{\prime * 2} m_{z}(6,0)+K_{1}^{\prime *} K_{2}^{\prime 3} m_{z}^{*}(6,0)
\end{aligned}
$$

with defining $K_{1}^{\prime}=K_{1}+w K_{2}^{*}$ and $K_{2}^{\prime}=K_{2}+w K_{1}^{*}$. Two solutions to $m_{y}(4,2)=0$ arise immediately: $K_{1}^{\prime}=0$ or $K_{2}^{\prime}=$ 0 , yielding

$$
w_{c, 1}=-\frac{K_{1}}{K_{2}^{*}} \quad \text { or } \quad w_{c, 2}=-\frac{K_{2}}{K_{1}^{*}}=w_{d}
$$

respectively. These are the same solutions as those found in [6] where the second-order circularity based techniques are applied. $w_{c, 2}$ is the same as the desired solution while $w_{c, 1}=$ $1 / w_{c, 2}^{*}$ is the so-called mirror solution. Given that $\left|K_{1}\right| \gg$ $\left|K_{2}\right|$, it is also evident that the desired solution always lies inside the unit circle, while the mirror solution is always in the outside.

According to the above analysis, as shown in (7), the signal does not satisfy sixth-order circular any more if the I/Q imbalance happens. Nevertheless, as shown in (10), the effects of I/Q imbalance can be effectively canceled by forcing the signal sixth-order circular again through adaptive filtering techniques. The method to update the coefficients of adaptive filter will be discussed in the following section.

\section{Newton's Method Applied to Compensation ESTIMATION}

Newton's method is well known in finding roots of equations by using the first few terms of the Taylor series of the function $f(w)$ in the vicinity of the suspected root. Its general form can be expressed as

$$
w_{k+1}=w_{k}-\frac{f\left(w_{k}\right)}{f^{\prime}\left(w_{k}\right)}
$$

where $k=0,1,2, \cdots$ is the iteration index, and the derivative of $f(w)$ with respect to $w=u+j v$ is defined as

$$
f^{\prime}(w)=\frac{\partial f(w)}{\partial w} \triangleq \frac{1}{2}\left(\frac{\partial}{\partial u}-j \frac{\partial}{\partial v}\right) f(u, v)
$$

Each zero of $f(w)$ has a domain of attraction, composed of the set of all starting points from which the algorithm converges to that particular zero. In the problem considered, under the assumption $\left|K_{1}\right| \gg\left|K_{2}\right|$, the desired solution is always close to the origin. This fact, coupled with the smooth (polynomial) behavior of the surface, indicates that the origin is always in the attraction domain of the desired solution. Therefore, starting the iteration from $w_{0}=0$ will always lead the Newton's algorithm as shown in (11) converge to the desired solution.

The I/Q imbalance compensation problem degrades into making the compensator output sixth-order circular, as shown in (8). Therefore, the compensation filter coefficient we wish to find is the root of the function $f(w) \triangleq m_{y}(4,2)=\mathrm{E}\left[y^{4} y^{* 2}\right]$. Inserting (5) into $m_{y}(4,2)$, based on the assumption that $z$ is sixth-order circular and $w$ is deterministic, the function $f(w)$ can be expressed through the sixth-order statistics of the imbalance signal $x$, as

$$
\begin{aligned}
f(w)= & w^{* 2} m_{x}(6,0)+\left(2 w^{*}+4 w^{*}|w|^{2}\right) m_{x}(5,1) \\
& +\left(1+8|w|^{2}+6|w|^{4}\right) m_{x}(4,2) \\
& +\left(4 w+12 w|w|^{2}+4 w|w|^{4}\right) m_{x}(3,3) \\
& +\left(6 w^{2}+8 w^{2}|w|^{2}+w^{2}|w|^{4}\right) m_{x}^{*}(4,2) \\
& +\left(4 w^{3}+2 w^{3}|w|^{2}\right) m_{x}^{*}(5,1)+w^{4} m_{x}^{*}(6,0)
\end{aligned}
$$

Calculating the derivative of $f(w)$ with respect to $w$, we get

$$
\begin{aligned}
f^{\prime}(w)= & 4 w^{* 2} m_{x}(5,1)+\left(8 w^{*}+12 w^{*}|w|^{2}\right) m_{x}(4,2) \\
& +\left(4+24|w|^{2}+12|w|^{4}\right) m_{x}(3,3) \\
& +\left(12 w+24 w|w|^{2}+4 w|w|^{4}\right) m_{x}^{*}(4,2) \\
& +\left(12 w^{2}+8 w^{2}|w|^{2}\right) m_{x}^{*}(5,1)+4 w^{3} m_{x}^{*}(6,0)
\end{aligned}
$$

All in sum, the solution can be found in a iterative form as shown in (11) by substituting $w \leftarrow w_{k}$ in (13) and (14), plugging these into (11), and starting the iteration from $w_{0}=$ 0.

On the other hand, due to the fact that the desired solution of $w_{d}$ is near the origin, hence, the right side of (14) can be approximated by $4 m_{x}(3,3)$. As a result, a simple stochastic form of Newton algorithm can be derived by approximating the expectation $\mathrm{E}\left[y^{4}(n) y^{* 2}(n)\right]$ with the instantaneous sample estimate and replacing the inverse of the derivative with a small positive constant $\mu$. Then we have

$$
\begin{gathered}
f\left(w_{n}\right) \leftarrow y^{4}(n) y^{* 2}(n) \\
\frac{1}{f^{\prime}\left(w_{n}\right)} \leftarrow \mu
\end{gathered}
$$

where $\mu$ is called the step size. Therefore, a single iteration of the stochastic Newton algorithm then reads

$$
\begin{gathered}
y(n)=x(n)+\hat{w}(n) x^{*}(n) \\
\hat{w}(n+1)=\hat{w}(n)-\mu y^{4}(n) y^{* 2}(n)
\end{gathered}
$$

Step size selection is a trade-off between the convergence speed and the steady-state variance of the algorithm.

\section{Simulations}

In this section, simulations are used to evaluate the performance of the proposed algorithms. In all the following simulations, the signal is a single-carrier 16-QAM waveform, and the imbalance parameters are $g=1.1$ and $\phi=10^{\circ}$, resulting in a front-end IRR of $20 \mathrm{~dB}$. The figure-of-merit is the post-compensation IRR, which is expressed as a function of the compensator estimate as [8]

$$
\operatorname{IRR}_{C}(\hat{w})=\frac{\mathrm{E}\left[\left|K_{1}+\hat{w} K_{2}^{*}\right|^{2}\right]}{\mathrm{E}\left[\left|K_{2}+\hat{w} K_{1}^{*}\right|^{2}\right]} \cong \frac{1}{\mathrm{E}\left[\left|e_{\hat{w}}\right|^{2}\right]}
$$




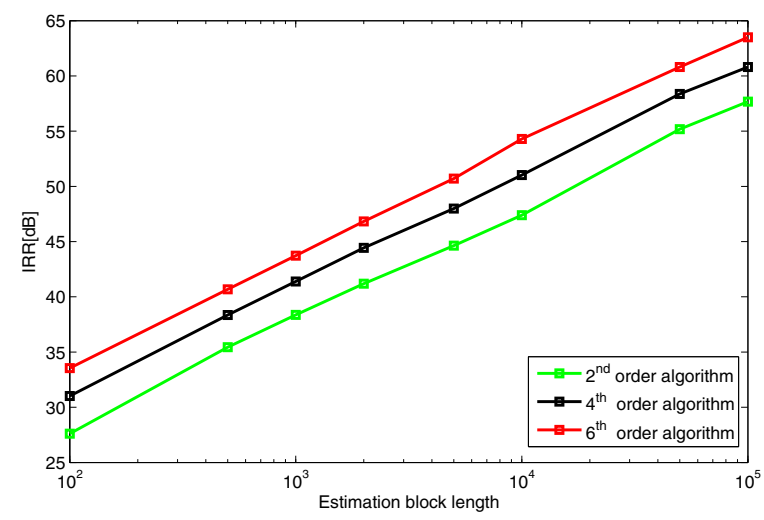

Fig. 3. The IRR of the tested block-form Newton algorithms versus the block length $L$ with $\mathrm{SNR}=20 \mathrm{~dB}$.

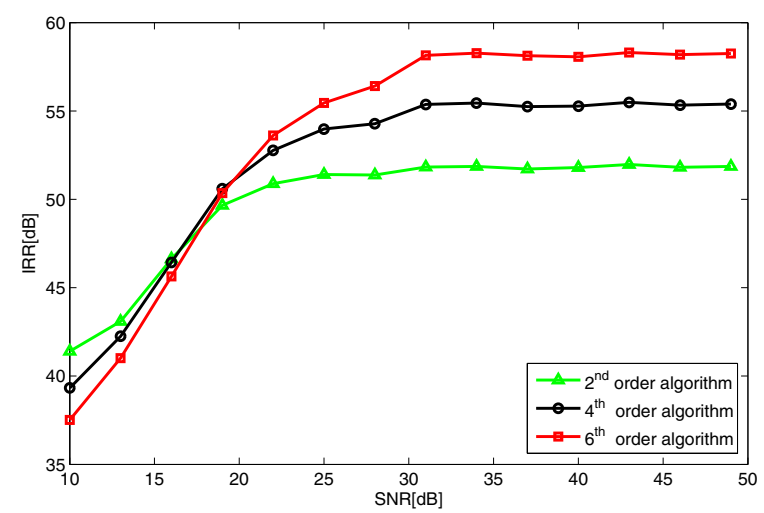

Fig. 4. The IRR of the tested block-form Newton algorithms versus the SNR with block length $L=10000$.

where $\mathrm{E}\left[\left|e_{\hat{w}}\right|^{2}\right]$ denotes the mean-square error of the compensator estimate.

The performances of the block-form Newton algorithm as shown in $(11,13,14)$ and its adaptive-form as shown in (1718) are compared with those of the corresponding secondorder and fourth-order circularity based algorithms from [6] and [8], respectively. These are the state-of-the-art blind I/Q calibration algorithms available in the literature. The blockform Newton algorithms are all iterated 10 times.

Fig. 3 shows the post-compensation IRR as a function of estimation block length $L$ with $\mathrm{SNR}=20 \mathrm{~dB}$ for the proposed sixth-order block-form algorithm and the baseline second-order and fourth-order block-form algorithms with 16QAM waveforms. Fig. 4 shows the post-compensation IRR as a function of SNR with a fixed block length $L=100000$ for the proposed sixth-order block-form algorithm and the reference second-order and fourth-order block-form algorithms with 16-QAM waveforms. In Fig. 3, the proposed sixthorder algorithm gives the highest image rejection value among the tested algorithms, followed by the fourth-order algorithm in [8] and the second-order algorithm in [6]. In Fig. 4, it can be observed that the second-order algorithm gives the

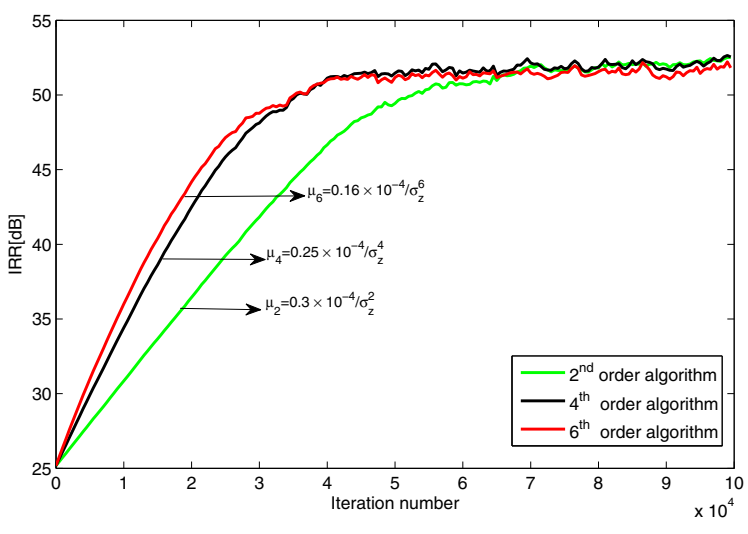

Fig. 5. Ensemble-average IRR of adaptive-form algorithms versus iteration number with 16-QAM signals in additive noise $(\mathrm{SNR}=20 \mathrm{~dB}) . \mu_{2}, \mu_{4}$ and $\mu_{6}$ are the step-size of the 2nd order, 4th order and 6th order algorithms, respectively, and $\sigma_{z}^{2}=\mathrm{E}\left[|\mathrm{z}|^{2}\right]$.

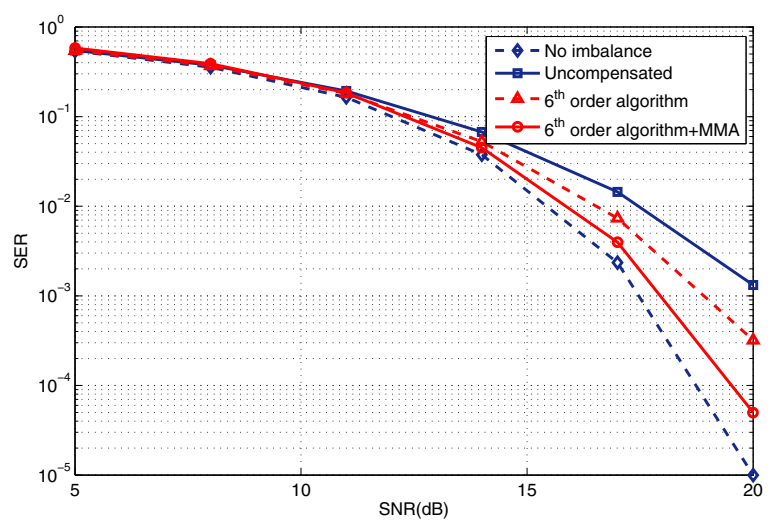

Fig. 6. Obtained SERs of the sixth-order algorithm with and without MMA in the detection of the single carrier 16-QAM signal.

highest image rejection value when the SNR is low (less than $17 \mathrm{~dB})$. However, as the SNR increases, the proposed sixthorder algorithm starts to outperform baseline algorithms and provides the highest image rejection value. The main reason is that the signal is more Gaussian when the SNR is low, while the statistics of an order higher than two can't provide any additional information.

We now compare the performances of the tested stochastic adaptive-form Newton algorithms. The simulation setup is the same as above, and the algorithms are iterated for 100,000 iterations. Various step-sizes are simulated, and the step-sizes are chosen such that the final steady-state performances of the two algorithms are equal. Fig. 5 shows the ensemble-averaged convergence curves, which demonstrates that the proposed sixth-order algorithm provides the fastest convergence rate, followed by the fourth-order algorithm and the second-order algorithm. This result is consistent with the result presented in Fig. 1, where the sixth-order statistic is shown to possess the steepest gradient with respect to the gain and phase imbalance factor. 
To compare the symbol error rate (SER) performance of stochastic adaptive algorithms, the simulation setup is the same as above, and the algorithms are iterated for 100,000 iterations. Fig. 6 shows the SER performances of the sixthorder algorithm with and without MMA. It is clear that the SER performance can be improved by using MMA eliminating the phase ambiguity.

\section{CONCLUSION}

In this paper, we have studied the feasibility of using higherorder circularity in receiver I/Q imbalance compensation. We showed that the moment $\mathrm{E}\left[\mathrm{z}^{4} \mathrm{z}^{* 2}\right]$ is a suitable measure of sixth-order circularity for a wide range of variables and signals encountered in communications. Moreover, we derived a general rule to choose the representative statistic for measuring the higher-order circularity. On the other hand, it is shown that the output of circularity-based I/Q compensator has phase ambiguity, which is necessary to eliminate. Then, two blind algorithms, based on the concept of sixth-order circularity and Newton's method, were proposed. One is based on block sample estimates of the sixth-order moments and the other is based on a stochastic adaptive learning rule. The methods were shown to outperform the state-of-the-art baseline methods which are based on second- and fourth-order circularity.

\section{ACKNOWLEDGMENT}

This work is supported in part by the Major Projects of the National Natural Science Foundation of China under Grant 91338105, and the foundation of Science and Technology on Information Transmission and Dissemination in Comm. Networks Lab.

\section{REFERENCES}

[1] A. Taighat, and A. H. Sayed, "Compensation schemes and performance analysis of I/Q imbalance in OFDM receivers," IEEE Transactions on Signal Processing, vol. 53, no. 8, pp. 3257-3268, 2005.

[2] K. Sung and C. Chao, "Estimation and compensation of I/Q imbalance in OFDM direct-conversion receivers," IEEE Journal of Selected Topics in Signal Processing, vol. 3, no. 3, pp. 438-453, 2009.

[3] Y. Chung and S. Phoong, "Joint estimation of I/Q imbalance, CFO and channel response for MIMO OFDM systems," IEEE Transactions on Communications, vol. 58, no. 5, pp. 1485-1492, 2010.

[4] G. Taubock, "Complex-valued random vectors and channels: Entropy, divergence, and capacity," IEEE Transactions on Information Theory, vol. 58, no. 5, pp. 2729-2744, 2012.

[5] M. Valkama, M. Renfors, and V. Koivunen, "Blind signal estimation in conjugate signal models with application to I/Q imbalance compensation," IEEE Signal Processing Letters, vol. 12, no. 11, pp. 733-736, 2005.

[6] L. Anttila, M. Valkama, and M. Renfors, "Circularity-based I/Q imbalance compensation in wideband direct-conversion receivers," IEEE Transactions on Vehicular Technology, vol. 57, no. 4, pp. 2099-2113, 2008.

[7] Y. Liu, R. Ranganathan, M. T. Hunter, W. B. Mikhael, and T. T. Yang, "Optimal block adaptive I/Q mismatch compensation based on circularity," in Proceedings of IEEE MWSCAS'10, Seattle, USA, pp. 320-323, 2010.

[8] L. Anttila, and M. Valkama, "Blind signal estimation in widely-linear signal models with fourth-order circularity: Algorithms and application to receiver I/Q calibration," IEEE Signal Processing Letters, vol. 20, no. 3, pp. 221-224, 2013
[9] M. Pedzisz, and D. P. Mandic, "Augmented complex statistics for signal prediction," Technical Report: TR-ICU-EPSRC08-07-TR002, Imperial College London, 2007. [online] http://www.commsp.ee.ic.ac.uk/mandic../research/Augumented_Statistic s_Report.pdf

[10] T. Adali, and P. Schreier, "Optimization and estimation of complexvalued signals: Theory and applications in filtering and blind source separation," IEEE Signal Processing Magazine, vol. 31, no. 5, pp. 112128, 2014.

[11] J. Yang, J. Werner, and G. A. Dumont, "The multimodulus blind equalization and its generalized algorithms," IEEE Journal on Selected Areas in Communications, vol. 20, no. 5, pp. 997-1015, 2002. 